\title{
Anomalous Hall effect in single-band chiral superconductors from impurity superlattices
}

\author{
Yu Li, ${ }^{1,2}$ Zhiqiang Wang $\odot,{ }^{3,4}$ and Wen Huang $\circledast^{1, *}$ \\ ${ }^{1}$ Shenzhen Institute for Quantum Science and Engineering \& Guangdong Provincial Key Laboratory of Quantum Science and Engineering, \\ Southern University of Science and Technology, Shenzhen 518055, Guangdong, China \\ ${ }^{2}$ Kavli Institute for Theoretical Sciences, University of Chinese Academy of Sciences, Beijing 100190, China \\ ${ }^{3}$ Department of Physics and Astronomy, McMaster University, Hamilton, Ontario, Canada L8S $4 M 1$ \\ ${ }^{4}$ James Franck Institute, University of Chicago, Chicago, Illinois 60637, USA
}

(Received 17 October 2019; revised 11 October 2020; accepted 28 October 2020; published 12 November 2020)

\begin{abstract}
Unlike anomalous quantum Hall insulators, clean single-band chiral superconductors do not exhibit intrinsic Hall effect at the one-loop approximation. Finite ac Hall conductance was found to emerge beyond one loop, such as with vertex corrections associated with extrinsic random impurity scatterings. In this paper, we investigate the effect of impurities embedded in single-band chiral superconductors in a superlattice pattern, instead of in random distributions. The impurity-induced Bogoliubov quasiparticle bound states hybridize to form subgap bands, constituting an emergent low-energy effective theory whose Hall effect can be studied with ease. We demonstrate that the occurrence of the Hall effect depends on the superlattice geometry and on the parity of the chiral pairing. In particular, due to the mixed particle-hole character of the subgap states, the Hall conductance may arise at the one-loop level of the current-current correlator in our effective model. Our theory provides an insight into the impurity-induced anomalous Hall effect in chiral superconductors.
\end{abstract}

DOI: 10.1103/PhysRevResearch.2.042027

Introduction. Topological chiral superconductors are classified by a topological invariant - the Chern number, and they exhibit protected chiral edge modes. Odd-parity chiral superconductors (e.g., chiral $p, f$ wave, etc.) may further support half-quantum vortices that host Majorana zero modes [1,2]. These excitations obey non-Abelian braiding statistics and could therefore be utilized for topological quantum computation [3-5].

The time-reversal symmetry breaking of the chiral pairings can be detected in polar Kerr effect measurements, where a linearly polarized light normally incident on the superconductor is reflected with a rotated polarization. Signatures of Kerr rotation have been reported in several unconventional superconductors, including $\mathrm{Sr}_{2} \mathrm{RuO}_{4}$ [6], $\mathrm{UPt}_{3}$ [7], and $\mathrm{URu}_{2} \mathrm{Si}_{2}$ [8]. Such an effect is closely related to the anomalous quantum Hall effect. However, unlike in an anomalous Hall insulator, the effect is not expected in a clean and uniform single-band chiral superconductor [2,9]. This could be understood in the following simple terms. The pairing potential $\Delta_{\mathbf{k}}$, whose $\mathbf{k}$ dependence describes the relative motion between the paired electrons, does not generate center-of-mass motion for the Cooper pair. Thus the current operator of a superconductor contains no contribution originating from $\Delta_{\mathbf{k}}$. Consequently, the Hall conductance is not directly related to the Berry curvature of the Bogoliubov quasiparticles and it in fact vanishes at the one-loop approximation.

\footnotetext{
*huangw3@sustech.edu.cn

Published by the American Physical Society under the terms of the Creative Commons Attribution 4.0 International license. Further distribution of this work must maintain attribution to the author(s) and the published article's title, journal citation, and DOI.
}

Nonetheless, vertex corrections, such as those arising from extrinsic impurity scatterings [9-14] and certain intrinsic superconducting collective modes [15-17], have both been shown to induce finite Hall conductance. Separate intrinsic mechanisms exist for multiband superconductors, but those involve interband Cooper pairing [18-22]. Thus far, whether these effects could quantitatively explain the observed Kerr rotation is still debated [23,24].

In previous studies, the leading order impurity effects are captured by the so-called skew-scattering diagrams [9,11-13]. However, these studies only capture the continuum state contribution, and some important microscopic details are absent in the diagrammatic treatment. In particular, individual impurities are known to induce subgap quasiparticle bound states $[25,26]$. How such low-energy states influence the electromagnetic response of the system remains largely unexplored and is the focus of the present study. In so doing, we uncover a different perspective on the impurity-induced Hall effect in chiral superconductors.

To facilitate our discussions, we imagine depositing impurities on the underlying chiral superconductors in a superlattice pattern (see Fig. 1). Due to the chiral nature of the pairing, the bound states from different impurity sites hybridize in a peculiar fashion that depends on their relative position. We construct a low-energy effective theory of the emergent subgap bands on the superlattice and study the resultant Hall response. Despite having similar appearance, the new effective Hamiltonian differs from the original BdG Hamiltonian in a fundamental way, that the components of the new spinor basis are no longer purely electron or hole, but rather a linear superposition of both. This mixed particle-hole character has profound consequences on transport properties, and we shall demonstrate finite Hall conductance at the one-loop level. 
(a) Square impurity superlattice

(b) Honeycomb impurity superlattice

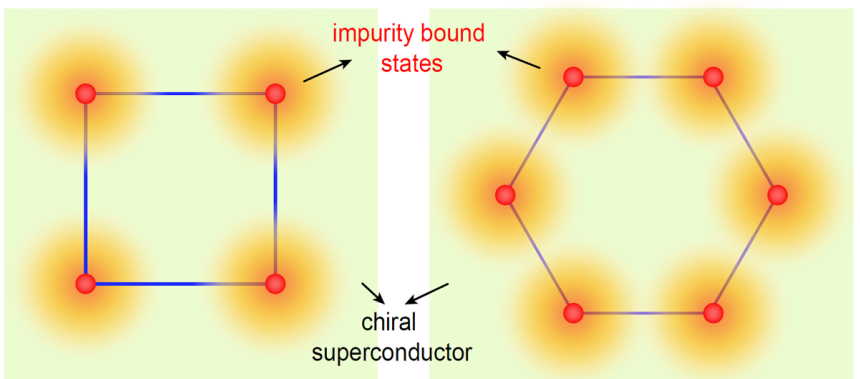

FIG. 1. (a) Square and (b) honeycomb impurity superlattices embedded in a chiral superconductor.

We consider several representative impurity superlattice geometries, and show that the resultant physics is model dependent. For example, while the Hall conductance is generically nonvanishing on a honeycomb superlattice in any underlying chiral pairing, it vanishes for square and triangular superlattices embedded in even-parity chiral superconductors, such as $d$ - and $g$-wave states.

Impurity states and impurity superlattice. In the Nambu spinor basis $\hat{\varphi}(\mathbf{r})=\left(c_{\mathbf{r} \uparrow}, c_{\mathbf{r} \downarrow}^{\dagger}\right)^{\top}$, the underlying single-band chiral superconducting state is described by the continuum Bogoliubov-de Gennes (BdG) Hamiltonian $H_{\mathrm{BdG}}^{\text {(bulk) }}=$ $\int d \mathbf{r} d \mathbf{r}^{\prime} \hat{\varphi}^{\dagger}(\mathbf{r}) \hat{H}_{\mathrm{BdG}}^{(\text {bulk })}\left(\mathbf{r}, \mathbf{r}^{\prime}\right) \hat{\varphi}\left(\mathbf{r}^{\prime}\right)+$ H.c., in which

$$
\hat{H}_{\mathrm{BdG}}^{\text {(bulk) }}\left(\mathbf{r}, \mathbf{r}^{\prime}\right)=\left(\begin{array}{cc}
\delta_{\mathbf{r}, \mathbf{r}^{\prime}}\left(-\frac{\nabla_{\mathbf{r}^{\prime}}}{2 m_{e}}-\mu\right) & \Delta\left(\mathbf{r}, \mathbf{r}^{\prime}\right) \\
\Delta^{*}\left(\mathbf{r}, \mathbf{r}^{\prime}\right) & \delta_{\mathbf{r}, \mathbf{r}^{\prime}}\left(\frac{\nabla_{\mathbf{r}^{\prime}}^{2}}{2 m_{e}}+\mu\right)
\end{array}\right),
$$

where $c_{\mathbf{r}}\left(c_{\mathbf{r}}^{\dagger}\right)$ stands for the electron annihilation (creation) operators, and $m_{e}$ and $\mu$ are the electron mass and the chemical potential, respectively. The off-diagonal term $\Delta\left(\mathbf{r}, \mathbf{r}^{\prime}\right)=$ $g\left(\left|\mathbf{r}-\mathbf{r}^{\prime}\right|\right) e^{i l \theta_{\mathbf{r}-\mathbf{r}^{\prime}}}$ is the chiral pairing potential, where $\theta_{\mathbf{r}}$ is the azimuthal angle of $\mathbf{r}$, and $g\left(\left|\mathbf{r}-\mathbf{r}^{\prime}\right|\right)$, assumed to be a certain (unimportant) decaying function of $\left|\mathbf{r}-\mathbf{r}^{\prime}\right|$, describes the spatial profile of the Cooper pair wave function. Here $l$ denotes the order of the chiral pairing, i.e., the Cooper pair angular momentum, which takes the values $1,2, \ldots$ for $p_{x}+$ $i p_{y}, d_{x^{2}-y^{2}}+i d_{x y}$ pairings, etc. Notice that we have assumed a uniform order parameter independent of the Cooper pair center-of-mass position, $\left(\mathbf{r}+\mathbf{r}^{\prime}\right) / 2$. Consideration of spatial variations around impurities does not qualitatively alter our conclusion.

Impurities in chiral superconductors are known to induce bound states. Consider first a single impurity at $\mathbf{R}=0$, described by a delta-function-like potential $U \delta(\mathbf{r}-\mathbf{R}) \tau_{3}$, where $U$ is the impurity strength and $\tau_{3}$ is the third component of the Pauli matrices operating in the Nambu space. The bound state wave functions take the forms $\psi_{+}(\mathbf{r})=[u(\mathbf{r}), v(\mathbf{r})]^{\top}=$ $\left(u_{r}, e^{-i l \theta_{\mathbf{r}}} v_{r}\right)^{\top}$ and $\psi_{-}(\mathbf{r})=\left[-v^{*}(\mathbf{r}), u^{*}(\mathbf{r})\right]^{\top}$ [27]. Here the "+" and "-" designate, respectively, the state with subgap energy $+E_{0}$ and the other with $-E_{0}$, where $E_{0}<\Delta_{0}$ and $\Delta_{0}$ denotes the superconducting gap. These two states are related by particle-hole symmetry, but the detailed forms of $u_{r}$ and $v_{r}$ are model dependent and are not constrained by any other symmetry, except that they shall in general decay as $e^{-r / \xi} / \sqrt{k_{\mathrm{F}} r}$ sufficiently far away from the impurity center. Here $k_{\mathrm{F}}$ is the Fermi momentum and $\xi$ the superconducting
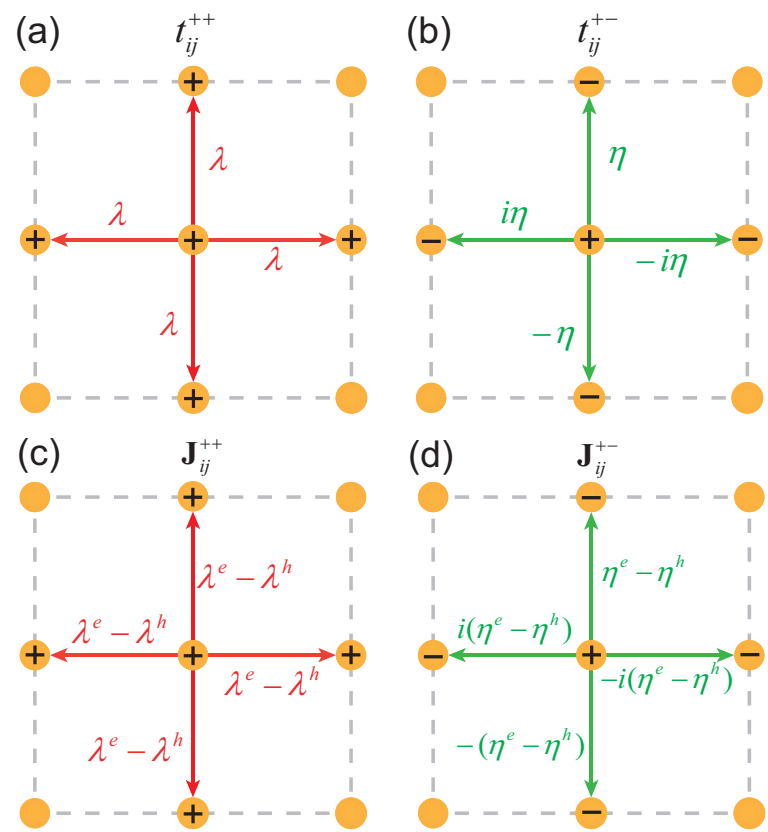

FIG. 2. (a),(b) Tight-binding construction of a square impurity superlattice immersed in a chiral $p$-wave superconductor. Note the relation $\lambda=\lambda^{\Delta}+\lambda^{e}+\lambda^{h}$ and $\eta=\eta^{\Delta}+\eta^{e}+\eta^{h}$. (c),(d) The current operator on the superlattice. The " + " and "-" symbols on the sites label the impurity bound states and arrows indicate the reference direction of hopping or current flow.

healing length. In the following, we shall assume a sizable impurity strength such that $E_{0} \ll \Delta_{0}$ [28,29], under which circumstance the low-energy theory associated with these bound states are well separated from the continuum spectrum.

On an impurity lattice where the interlattice spacing $R_{0}$ is larger than $\xi$, the above-stated bound state wave functions on each single site still constitute a good approximation. States from neighboring impurity sites "hybridize" via the microscopic kinetic hopping and pairing in the original Hamiltonian Eq. (1). Written in the second quantized form where $c_{i, \pm}^{\dagger}\left(c_{i, \pm}\right)$ denote the creation (annihilation) of the respective bound states on each site, an emergent lowenergy tight-binding model on the superlattice reads $H^{\text {eff }}=$ $\sum_{i, j} \hat{\Psi}_{i}^{\dagger}\left[E_{0} \delta_{i j} \sigma_{3}+\hat{h}_{i j}\left(1-\delta_{i j}\right)\right] \hat{\Psi}_{j}+$ H.c., with the Pauli $\sigma$ matrices operating in the space spanned by $\hat{\Psi}_{i}=\left(c_{i,+}, c_{i,-}\right)^{\mathrm{T}}$, and

$$
\hat{h}_{i j}=\left(\begin{array}{cc}
t_{i j}^{++} & t_{i j}^{+-} \\
t_{i j}^{-+} & t_{i j}^{--}
\end{array}\right),
$$

in which

$$
t_{i j}^{\mu \nu}=\int d \mathbf{r} d \mathbf{r}^{\prime} \psi_{\mu}^{\dagger}\left(\mathbf{r}-\mathbf{R}_{i}\right) \hat{H}_{\mathrm{BdG}}\left(\mathbf{r}, \mathbf{r}^{\prime}\right) \psi_{\nu}\left(\mathbf{r}^{\prime}-\mathbf{R}_{j}\right) .
$$

It is obvious that the hopping of the bound states could arise from both the kinetic and pairing terms in the underlying microscopic Hamiltonian. A detailed analysis of the hopping integrals can be found in the Supplemental Material [27], which we summarize below and in Figs. 2(a) and 2(b). The hybridization between the $+(-)$ states satisfy $t_{i j}^{++}=$ $-t_{i j}^{--}=\lambda_{i j}$, where $\lambda_{i j}$ is a real constant determined by the separation $\left|\mathbf{R}_{j}-\mathbf{R}_{i}\right|$. On the other hand, the integral between 
the + and - states has the relation $t_{i j}^{+-}=\left(t_{i j}^{-+}\right)^{*}=\eta_{i j}$, where $\eta_{i j}=\left|\eta_{i j}\right| e^{i l \theta_{\mathbf{R}_{j}-\mathbf{R}_{i}}}$ [30]. It thus depends on both the relative position between the two sites and the order of the chiral pairing.

Our later analyses of the current operators require distinguishing in Eq. (3) contributions originating from the pairing, and the electron and hole kinetic hopping processes, i.e., $\lambda_{i j}=$ $\lambda_{i j}^{\Delta}+\lambda_{i j}^{e}+\lambda_{i j}^{h}$ and $\eta_{i j}=\eta_{i j}^{\Delta}+\eta_{i j}^{e}+\eta_{i j}^{h}$. The kinetic part of $\eta_{i j}, \eta_{i j}^{e}+\eta_{i j}^{h}$, deserves special attention. Written explicitly,

$$
\begin{aligned}
\eta_{i j}^{e}+\eta_{i j}^{h} & =\int d \mathbf{r} d \mathbf{r}^{\prime}\left[-u_{\left|\mathbf{r}-\mathbf{R}_{i}\right|} \delta_{\mathbf{r}, \mathbf{r}^{\prime}}\left(-\nabla_{\mathbf{r}^{\prime}}^{2} / 2 m_{e}-\mu\right) e^{i l \theta_{\mathbf{r}^{\prime}-\mathbf{R}_{j}}} v_{\left|\mathbf{r}^{\prime}-\mathbf{R}_{j}\right|}+e^{i l \theta_{\mathbf{r}-\mathbf{R}_{i}}} v_{\left|\mathbf{r}-\mathbf{R}_{i}\right|} \delta_{\mathbf{r}^{\prime}, \mathbf{r}}\left(\nabla_{\mathbf{r}^{\prime}}^{2} / 2 m_{e}+\mu\right) u_{\left|\mathbf{r}^{\prime}-\mathbf{R}_{j}\right|}\right] \\
& =\left(1+e^{i l \pi}\right) \int d \tilde{\mathbf{r}} d \tilde{\mathbf{r}}^{\prime} u_{\tilde{r}} \delta_{\tilde{\mathbf{r}}, \tilde{\mathbf{r}}^{\prime}-\left(\mathbf{R}_{i}-\mathbf{R}_{j}\right)}\left(\nabla_{\tilde{\mathbf{r}}^{\prime}}^{2} / 2 m_{e}+\mu\right) e^{i l \theta_{\tilde{\mathbf{r}}^{\prime}}} v_{\tilde{r}^{\prime}},
\end{aligned}
$$

where we have performed partial integration and substitution of variables to obtain the second line, and the two terms in $1+e^{i l \pi}$ are associated with $\eta_{i j}^{e}$ and $\eta_{i j}^{h}$, respectively. The relation $\left|\eta_{i j}^{e}\right|=\left|\eta_{i j}^{h}\right|$ is a consequence of the particle-hole symmetry between the + and - states. For $l$ odd, $\eta_{i j}^{e}=-\eta_{i j}^{h}$; hence the kinetic contribution vanishes if the underlying chiral pairing has odd parity; for $l$ even, by contrast, $\eta_{i j}^{e}=\eta_{i j}^{h}$. We shall later see that the corresponding current operators have the opposite even and odd $l$ dependence. Finally, it is easy to check that the relation $\eta_{i j}=\left|\eta_{i j}\right| e^{i l \theta_{\mathbf{R}_{j}-\mathbf{R}_{i}}}$ also holds for the individual constituents of $\eta_{i j}$.

As a concrete example, in a chiral $p$-wave superconductor, a square impurity superlattice with up to nearest-neighbor hopping has the following effective Hamiltonian:

$$
\hat{H}_{\mathbf{k}}^{\text {eff }}=\mathcal{E}_{3 \mathbf{k}} \sigma_{3}+\mathcal{E}_{1 \mathbf{k}} \sigma_{1}-\mathcal{E}_{2 \mathbf{k}} \sigma_{2},
$$

where we have set $R_{0}=1$ for brevity, $\mathcal{E}_{3 \mathbf{k}}=2 \lambda\left(\cos k_{x}+\right.$ $\left.\cos k_{y}\right)+E_{0}, \mathcal{E}_{1 \mathbf{k}}=2 \eta \sin k_{x}$, and $\mathcal{E}_{2 \mathbf{k}}=2 \eta \sin k_{y}$. Here, $\lambda$ denotes the nearest-neighbor hopping integrals of $\lambda_{i j}$ and $\eta$ the corresponding counterpart of $\left|\eta_{i j}\right|$. Notice the implicit decomposition such as $\eta=\eta^{\Delta}+\eta^{e}+\eta^{h}$ (although $\eta^{e}+\eta^{h}=0$ for chiral $p$ wave). Due to the angle dependence of the complex off-diagonal hopping $\eta_{i j}$, Eq. (5) resembles the form of the underlying chiral $p$ wave Hamiltonian. The band topology could be engineered by controlling parameters such as the impurity potential and the superlattice constant $[29,31]$. These hold for higher order chiral superconductors, although further neighbor hybridizations must be considered to make the band topology transparent. In like manner, impurity chains immersed in odd-parity chiral states support an emergent 1D $p$-wave model and may give rise to isolated Majorana zero modes at the ends of the chains.

Current operators. The mixed particle-hole nature of each of the spinor component in $\hat{\Psi}$ (i.e., each impurity bound state) has a profound consequence on the particle current operators. Foremost, the portion of the hopping integrals originating from the underlying Cooper pairing, i.e., $\lambda^{\Delta}$ and $\eta^{\Delta}$, shall have no contribution, as in the case of clean superconductors. The only contribution stems from the mutually "canceling" electron hopping $\left(\lambda^{e}\right.$ and $\left.\eta^{e}\right)$ and hole hopping $\left(\lambda^{h}\right.$ and $\left.\eta^{h}\right)$. Understandably, if the + state is purely electronlike and the state purely holelike, $\eta^{e}=\eta^{h}=0$, and the resultant current operators resemble those of a clean superconductor.

For the model given in (5), the current operators $\mathbf{J}_{i j}^{++}$ and $\mathbf{J}_{i j}^{+-}$defined on the superlattice bonds are sketched in
Figs. 2(c) and 2(d). The properties of the $t_{i j}^{\mu \nu}$ 's imply the following general relation: $\mathbf{J}_{i j}^{++}=-\left(\mathbf{J}_{i j}^{--}\right)^{*}$ and $\mathbf{J}_{i j}^{+-}=\left(\mathbf{J}_{i j}^{-+}\right)^{*}$. Specific to the model in (5), the $x$ component of the current operator reads

$$
\hat{J}_{x \mathbf{k}}^{\text {eff }}=\mathcal{J}_{3 x \mathbf{k}} \sigma_{3}+\mathcal{J}_{1 x \mathbf{k}} \sigma_{1}+\mathcal{J}_{2 x \mathbf{k}} \sigma_{2},
$$

where $\mathcal{J}_{3 x \mathbf{k}}=-2\left(\lambda^{e}-\lambda^{h}\right) \sin k_{x}, \mathcal{J}_{1 x \mathbf{k}}=2\left(\eta^{e}-\eta^{h}\right) \cos k_{x}$, and $\mathcal{J}_{2 x \mathbf{k}}=0$. Note that $\mathcal{J}_{2 x}$ could be nonzero if further neighbor hoppings are considered. The $y$ component follows similarly and can be found in the Supplemental Material [27]. The cancellation between the electron and hole contributions is evident in these expressions. Notably, although $\eta^{e}+\eta^{h}=0$ for odd-parity pairing, the corresponding kinetic contribution to the particle current is finite and scales as $\eta^{e}-\eta^{h}=2 \eta^{e}$, such as in $\mathcal{J}_{1 x \mathbf{k}}$. In the case of underlying even-parity pairing, however, since $\eta^{e}=\eta^{h}, \mathbf{J}_{i j}^{+-} \propto \eta^{e}-\eta^{h}=0$ - suggesting a perfect cancellation between the electron and hole transport. Hence $\mathcal{J}_{1 x(y)}$ and $\mathcal{J}_{2 x(y)}$ must both vanish in this case.

Anomalous Hall conductivity. We are now in position to study the anomalous Hall conductance of our low-energy theory. Within linear response theory, it is given by the antisymmetric part of the $\hat{J}_{x}-\hat{J}_{y}$ correlation function $\pi_{x y}(\mathbf{q}, \omega)$,

$$
\sigma_{\mathrm{H}}(\omega)=\frac{i}{2 \omega} \lim _{\mathbf{q} \rightarrow 0}\left[\pi_{x y}(\mathbf{q}, \omega)-\pi_{y x}(\mathbf{q}, \omega)\right],
$$

where, at the one-loop approximation,

$\pi_{x y}\left(\mathbf{q}=0, i v_{m}\right)=T \sum_{\mathbf{k}, i \omega_{n}} \operatorname{Tr}\left[\hat{J}_{x \mathbf{k}}^{\mathrm{eff}} \hat{G}\left(\mathbf{k}, i \omega_{n}+i v_{m}\right) \hat{J}_{y \mathbf{k}}^{\mathrm{eff}} \hat{G}\left(\mathbf{k}, i \omega_{n}\right)\right]$,

where $T$ is the temperature, $\omega_{n}=(2 n+1) \pi T$ and $v_{m}=2 m \pi T$ are, respectively, the fermionic and bosonic Matsubara frequencies, and $\hat{G}\left(\mathbf{k}, i \omega_{n}\right)=\left(i \omega_{n}-\hat{H}_{\mathbf{k}}^{\text {eff }}\right)^{-1}$ stands for the impurity-band Green's function. For the square lattice model introduced above, we arrive at the following:

$$
\sigma_{\mathrm{H}}(\omega+i \delta)=\sum_{\mathbf{k}} \frac{f_{\mathbf{k}}}{E_{\mathbf{k}}\left[(\omega+i \delta)^{2}-4 E_{\mathbf{k}}^{2}\right]},
$$

where $E_{\mathbf{k}}=\sqrt{\mathcal{E}_{1 \mathbf{k}}^{2}+\mathcal{E}_{2 \mathbf{k}}^{2}+\mathcal{E}_{3 \mathbf{k}}^{2}}$ is the dispersion of the impurity subgap band, and

$$
f_{\mathbf{k}}=\sum_{m, n, s} \frac{\epsilon^{m n s}}{2}\left[\mathcal{J}_{m x \mathbf{k}} \mathcal{J}_{n y \mathbf{k}}-\mathcal{J}_{m y \mathbf{k}} \mathcal{J}_{n x \mathbf{k}}\right] \mathcal{E}_{\mathbf{s k}},
$$


TABLE I. Anomalous Hall effect in representative continuum chiral superconductors generated by impurity subgap bands emerging from various embedding impurity configurations. In comparison, the last row presents the prediction of the diagrammatic skewscattering approach, which only considers the continuum state contribution $[9,11]$

\begin{tabular}{lcccc}
\hline \hline $\begin{array}{l}\text { Superlattice } \\
\text { structure }\end{array}$ & $\begin{array}{l}p \text { wave } \\
(l=1)\end{array}$ & $\begin{array}{l}d \text { wave } \\
(l=2)\end{array}$ & $\begin{array}{c}f \text { wave } \\
(l=3)\end{array}$ & $\begin{array}{c}g \text { wave } \\
(l=4)\end{array}$ \\
\hline Square & $\checkmark$ & $\times$ & $\checkmark$ & $\times$ \\
Triangular & $\checkmark$ & $\times$ & $\checkmark$ & $\times$ \\
Honeycomb & $\checkmark$ & $\checkmark$ & $\checkmark$ & $\checkmark$ \\
Random (continuum) & $\checkmark$ & $\times$ & $\times$ & $\times$ \\
\hline \hline
\end{tabular}

where $\epsilon^{m n s}$ denotes the Levi-Civita tensor with indices $m, n, s=1,2,3$. Obviously, $\sigma_{\mathrm{H}}$ vanishes for any underlying even-parity chiral pairing, as their current operators contain only $\mathcal{J}_{3 x(y)}$, even when further neighbor hoppings are included. In contrast, odd-parity pairings shall in general see a finite Hall conductance. This distinction applies to any superlattice configuration with no sublattice degree of freedom, including triangular lattices (see Table I and Ref. [27]).

There are several features worth remarking. First, the magnitude of $\sigma_{\mathrm{H}}$ is determined by the above-defined hopping integrals which describe the hybridization between the impurity-bound states. Since these parameters overall grow exponentially with decreasing impurity spacing, one expects the conductivity to enhance exponentially with increasing impurity concentration. This contrasts with the skew-scattering diagrammatic analysis which captures only the continuum state contributions $[9,11]$. Secondly, the cutoff frequency at which the imaginary part of $\sigma_{\mathrm{H}}$ becomes nonzero (where a sharp peak appears) is set by the gap between the impurity bands. By contrast, continuum state contributions cut off at $w=2 \Delta_{0}[9,11]$. Finally, unlike the proposals which require particle-hole asymmetric normal state electron dispersion to obtain finite $\sigma_{\mathrm{H}}[9,18]$, our low-energy theory has no such restriction.

Honeycomb superlattice. The mixed particle-hole nature of the impurity subgap bands implies that there exists no fundamental symmetry constraints to prohibit the Hall effect in our effective theory. In other words, the vanishing of $\sigma_{\mathrm{H}}$ in some of the models above must be accidental. Given that those models are characterized by single-sigma-matrix current operators, looking for systems that exhibit more structured current operators may be a promising route to obtain finite $\sigma_{\mathrm{H}}$. One possibility is to introduce sublattice degrees of freedom. We verify this conjecture through a honeycomb lattice model [Fig. 1(b)].

Consider up to nearest-neighbor terms, in the basis $\Psi_{i}=$ $\left(c_{i,+}, c_{i,+}^{\prime}, c_{i,-}, c_{i,-}^{\prime}\right)^{\top}$, where $c$ and $c^{\prime}$ represent the two sublattices, the emergent tight-binding Hamiltonian has the form [27],

$$
\hat{H}_{\mathbf{k}}^{\text {eff }}=\left[\begin{array}{cccc}
E_{0} & \lambda_{\mathbf{k}} & 0 & \eta_{\mathbf{k}} \\
\lambda_{\mathbf{k}}^{*} & E_{0} & (-1)^{l} \eta_{-\mathbf{k}} & 0 \\
0 & (-1)^{l} \eta_{-\mathbf{k}}^{*} & -E_{0} & -\lambda_{\mathbf{k}} \\
\eta_{\mathbf{k}}^{*} & 0 & -\lambda_{\mathbf{k}}^{*} & -E_{0}
\end{array}\right],
$$

where $\quad \lambda_{\mathbf{k}}=\sum_{\delta} e^{i \mathbf{k} \cdot \overline{\mathbf{R}}_{\delta}} \lambda \quad$ and $\quad \eta_{\mathbf{k}}=\sum_{\delta} e^{i \mathbf{k} \cdot \overline{\mathbf{R}}_{\delta}} e^{i l \theta_{\overline{\mathbf{R}}_{\delta}} \eta}$, and $\overline{\mathbf{R}}_{\delta}(\delta=1,2,3)$ designate the three shortest vectors connecting sublattice $c$ to $c^{\prime}$. Interestingly, at $E_{0}=0$, the model resembles a low-energy theory proposed for the Moiré superlattice in twisted bilayer graphene [32].

As we have seen, in the case of even-parity pairing, the hopping between the + and - states on different sites does not generate particle current. However, the intersublattice hopping between the $+($ or - ) states introduces two off-diagonal components in the current operators. For example, in the present model,

$$
\hat{J}_{x \mathbf{k}}^{\text {eff }}=\mathcal{J}_{1 x \mathbf{k}} \varrho_{1} \otimes \sigma_{3}+\mathcal{J}_{2 x \mathbf{k}} \varrho_{2} \otimes \sigma_{3},
$$

in which $\varrho_{i}(i=1,2,3)$ are the Pauli matrices operating in the sublattice manifold, and $\mathcal{J}_{1 x \mathbf{k}}=-3\left(\lambda^{e}-\lambda^{h}\right)$ $\sin \left(\frac{3 k_{x}}{2}\right) \cos \left(\frac{\sqrt{3} k_{y}}{2}\right)$ and $\mathcal{J}_{2 x \mathbf{k}}=3\left(\lambda^{e}-\lambda^{h}\right) \cos \left(\frac{3 k_{x}}{2}\right) \cos \left(\frac{\sqrt{3} k_{y}}{2}\right)$. A lengthy calculation for $\sigma_{\mathrm{H}}(w)$ presented in the Supplemental Material [27] leads to an integral form involving $\left[\mathcal{J}_{1 x \mathbf{k}} \mathcal{J}_{2 y \mathbf{k}}-\mathcal{J}_{2 x \mathbf{k}} \mathcal{J}_{1 y \mathbf{k}}\right]\left(\left|\eta_{\mathbf{k}}\right|^{2}-\left|\eta_{-\mathbf{k}}\right|^{2}\right) E_{0}$ in the numerator of the integrand. The integral is generically finite, in contrast to the square and triangular superlattice scenarios. For oddparity pairings, an additional contribution to the current operators arises from the intersublattice hopping between the + and - states, and the Hall conductance is again finite.

Concluding remarks. Table I summarizes our main results and makes a comparison to the conclusion obtained from the skew-scattering diagrammatic calculations [11]. Since the latter approach had only accounted for contributions from the continuum states, our theory suggests that random impurities still have the potential to induce a finite Hall response in higher-order chiral superconducting states if the impurityinduced subgap states are considered.

Current scanning tunneling microscopy techniques have enabled atomically controlled defect engineering [33], paving the way for studying the Hall effect in chiral superconductors with any desired impurity configuration. Our theory also suggests a viable means to probe charge-neutral chiral superfluids in liquid helium-3 [34,35] and in trapped cold Fermi gases [36-38]. In these systems, ordered defects could be prepared using a periodically modulated holder potential or by shining patterned laser beams. In the case of quantum gases, for example, the resultant conductivity may be obtained by measuring the response of the system to a time-dependent trapping potential [39-42].

In summary, we have provided an alternative perspective on the impurity-induced anomalous Hall response in chiral superconductors. Previous studies of this kind had relied exclusively on a diagrammatic approach involving extensive vertex correction analyses, and they had only accounted for the contribution from the quasiparticle continuum. By laying our eyes on the effect of impurity induced bound states, we constructed an emergent low energy theory for when the impurities are deposited in superlattice patterns. Owing to the mixed particle-hole character of the bound states, the resultant theory generates finite Hall conductivity at the oneloop level of the approximation-without the need for vertex corrections. 
Acknowledgments. We acknowledge fruitful discussions with F.-C. Zhang. This work is supported by NSFC under Grant No. 11904155 (W.H.), the Guangdong Provincial Key
Laboratory under Grant No. 2019B121203002 (W.H.), and the China Postdoctoral Science Foundation under Grant No. 2020M670422 (Y.L.).
[1] G. E. Volovik, Fermion zero modes on vortices in chiral superconductors, JETP Lett. 70, 609 (1999).

[2] N. Read and D. Green, Paired states of fermions in two dimensions with breaking of parity and time-reversal symmetries and the fractional quantum Hall effect, Phys. Rev. B 61, 10267 (2000).

[3] D. A. Ivanov, Non-Abelian Statistics of Half-Quantum Vortices in $p$-Wave Superconductors, Phys. Rev. Lett. 86, 268 (2001).

[4] A. Yu. Kitaev, Fault-tolerant quantum computation by anyons, Ann. Phys. (NY) 303, 2 (2003).

[5] C. Nayak, S. H. Simon, A. Stern, M. Freedman, and S. Das Sarma, Non-Abelian anyons and topological quantum computation, Rev. Mod. Phys. 80, 1083 (2008).

[6] J. Xia, Y. Maeno, P. T. Beyersdorf, M. M. Fejer, and A. Kapitulnik, High Resolution Polar Kerr Effect Measurements of $\mathrm{Sr}_{2} \mathrm{RuO}_{4}$ : Evidence for Broken Time-Reversal Symmetry in the Superconducting State, Phys. Rev. Lett. 97, 167002 (2006).

[7] E. R. Schemm, W. J. Gannon, C. M. Wishne, W. P. Halperin, and A. Kapitulnik, Observation of broken time-reversal symmetry in the heavy-fermion superconductor $\mathrm{UPt}_{3}$, Science 345, 190 (2014).

[8] E. R. Schemm, R. E. Baumbach, P. H. Tobash, F. Ronning, E. D. Bauer, and A. Kapitulnik, Evidence for broken time-reversal symmetry in the superconducting phase of $\mathrm{URu}_{2} \mathrm{Si}_{2}$, Phys. Rev. B 91, 140506(R) (2015).

[9] R. M. Lutchyn, P. Nagornykh, and V. M. Yakovenko, Frequency and temperature dependence of the anomalous ac Hall conductivity in a chiral $p_{x}+i p_{y}$ superconductor with impurities, Phys. Rev. B 80, 104508 (2009).

[10] N. A. Sinitsyn, Semiclassical theories of the anomalous Hall effect, J. Phys.: Condens. Matter 20, 023201 (2008).

[11] J. Goryo, Impurity-induced polar Kerr effect in a chiral $p$-wave superconductor, Phys. Rev. B 78, 060501(R) (2008).

[12] J. Goryo, Intrinsic and extrinsic origins of the polar Kerr effect in a chiral $p$-wave superconductors, Mod. Phys. Lett. B 24, 2831 (2010).

[13] E. J. König and A. Levchenko, Kerr Effect from Diffractive Skew Scattering in Chiral $p_{x} \pm i p_{y}$ Superconductors, Phys. Rev. Lett. 118, 027001 (2017).

[14] S. Li, A. V. Andreev, and B. Z. Spivak, Anomalous transport phenomena in $p_{x}+i p_{y}$ superconductors, Phys. Rev. B 92, 100506(R) (2015).

[15] S. K. Yip and J. A. Sauls, Circular dichroism and birefringence in unconventional superconductors, J. Low Temp. Phys. 86, 257 (1992).

[16] R. Roy and C. Kallin, Collective modes and electromagnetic response of a chiral superconductor, Phys. Rev. B 77, 174513 (2008).

[17] R. M. Lutchyn, P. Nagornykh, and V. M. Yakovenko, Gaugeinvariant electromagnetic response of a chiral $p_{x}+i p_{y}$ superconductor, Phys. Rev. B 77, 144516 (2008).
[18] E. Taylor and C. Kallin, Intrinsic Hall Effect in a Multiband Chiral Superconductor in the Absence of an External Magnetic Field, Phys. Rev. Lett. 108, 157001 (2012).

[19] K. I. Wysokinski, J. F. Annett, and B. L. Gyorffy, Intrinsic Optical Dichroism in the Chiral Superconducting State of $\mathrm{Sr}_{2} \mathrm{RuO}_{4}$, Phys. Rev. Lett. 108, 077004 (2012).

[20] Z. Wang, J. Berlinsky, G. Zwicknagl, and C. Kallin, Intrinsic ac anomalous Hall effect of nonsymmorphic chiral superconductors with an application to $\mathrm{UPt}_{3}$, Phys. Rev. B 96, 174511 (2017).

[21] P. M. R. Brydon, D. S. L. Abergel, D. F. Agterberg, and V. M. Yakovenko, Loop Currents and Anomalous Hall Effect from Time-Reversal Symmetry-Breaking Superconductivity on the Honeycomb Lattice, Phys. Rev. X 9, 031025 (2019).

[22] L. Komendová and A. M. Black-Schaffer, Odd-Frequency Superconductivity in $\mathrm{Sr}_{2} \mathrm{RuO}_{4}$ Measured by Kerr Rotation, Phys. Rev. Lett. 119, 087001 (2017).

[23] C. Kallin and A. J. Berlinsky, Chiral superconductors, Rep. Prog. Phys. 79, 054502 (2016).

[24] K. I. Wysokiński, Time reversal symmetry breaking superconductors: $\mathrm{Sr}_{2} \mathrm{RuO}_{4}$ and beyond, Condens. Matter 4, 47 (2019).

[25] Y. Okuno, M. Matsumoto, and M. Sigrist, Analysis of impurity induced circular currents for the chiral superconductor $\mathrm{Sr}_{2} \mathrm{RuO}_{4}$, J. Phys. Soc. Jpn 68, 3054 (1999).

[26] A. V. Balatsky, I. Vekhter, and J.-X. Zhu, Impurity-induced states in conventional and unconventional superconductors, Rev. Mod. Phys. 78, 373 (2006).

[27] See the Supplemental Material at http://link.aps.org/ supplemental/10.1103/PhysRevResearch.2.042027 for the derivation of the impurity bound state solutions, the derivation of the hopping integrals between the impurity bound states and their symmetry properties, more detailed construction of the effective tight-binding models and their current operators, and the calculation of the Hall conductance in square and honeycomb impurity superlattices.

[28] V. Kaladzhyan, C. Bena, and P. Simon, Characterizing p-wave superconductivity using the spin structure of Shiba states, Phys. Rev. B 93, 214514 (2016).

[29] V. Kaladzhyan, J. Röntynen, P. Simon, and T. Ojanen, Topological state engineering by potential impurities on chiral superconductors, Phys. Rev. B 94, 060505(R) (2016).

[30] Notice that the form of this hybridization is locked with the $U(1)$ phase of the underlying chiral pairing. For example, for chiral pairings with $\Delta_{\mathbf{k}} \sim\left(k_{x}+i k_{y}\right)^{l}$ and $\Delta_{\mathbf{k}} \sim e^{i \varphi}\left(k_{x}+i k_{y}\right)^{l}$, their $\eta_{i j}$ must differ by the same phase $\varphi$.

[31] L. Kimme and T. Hyart, Existence of zero-energy impurity states in different classes of topological insulators and superconductors and their relation to topological phase transition, Phys. Rev. B 93, 035134 (2016).

[32] N. F. Q. Yuan and L. Fu, Model for the metal-insulator transition in graphene superlattices and beyond, Phys. Rev. B 98, 045103 (2018). 
[33] A. A. Khajetoorians, D. Wegner, A. F. Otte, and I. Swart, Creating designer quantum states of matter atom-by-atom, Nat. Rev. Phys. 1, 703 (2019).

[34] P. W. Anderson and P. Moreal, Generalized Bardeen-CooperSchrieffer states and the proposed low-temperature phase of liquid $\mathrm{He}^{3}$, Phys. Rev. 123, 1911 (1961).

[35] P. W. Anderson and W. F. Brinkman, Anisotropic Superfluidity in ${ }^{3} \mathrm{He}$ : A Possible Interpretation of its Stability as a SpinFluctuation Effect, Phys. Rev. Lett. 30, 1108 (1973).

[36] B. Liu, X. Li, B. Wu, and W. V. Liu, Chiral superfluidity with $p$-wave symmetry from an interacting $s$-wave atomic Fermi gas, Nat. Commun. 5, 5064 (2014).

[37] A. Bühler, N. Lang, C. V. Kraus, G. Möller, S. D. Huber, and H. P. Büchler, Majorana modes and $p$-wave superfluids for fermionic atoms in optical lattices, Nat. Commun. 5, 4504 (2014).
[38] Z. Wu and G. M. Bruun, Topological Superfluid in a FermiBose Mixture with a High Critical Temperature, Phys. Rev. Lett. 117, 245302 (2016).

[39] Z. Wu, E. Taylor, and E. Zaremba, Probing the optical conductivity of trapped charge-neutral quantum gases, Europhys. Lett. 110, 26002 (2015).

[40] J. M. Midtgaard, Z. Wu, N. Goldman, and G. M. Bruun, Detecting chiral pairing and topological superfluidity using circular dichroism, Phys. Rev. Research 2, 033385 (2020).

[41] J. Röntynen and T. Ojanen, Topological Superconductivity and High Chern Numbers in 2D Ferromagnetic Shiba Lattices, Phys. Rev. Lett. 114, 236803 (2015).

[42] L. H. Santos, Y. Wang, and E. Fradkin, Pair-Density-Wave Order and Paired Fractional Quantum Hall Fluids, Phys. Rev. X 9, 021047 (2019). 